Boletín de la Sociedad Zoológica del Uruguay, 2020

Vol. 29 (2): 187-192

ISSN 2393-6940

https://journal.szu.org.uy

\title{
NOTA
}

\section{ZAMBA POR VOS: POSIBLE ESTRIDULACIÓN DURANTE EL CORTEJO EN UNA ARAÑA CON VELO NUPCIAL (CTENIDAE)}

\author{
Mariana Celeste Trillo1, 2, Álvaro Laborda², Gabriel Francescoli³ \& Anita Aisenberg ${ }^{1}$ \\ 1 Departamento de Ecología y Biología Evolutiva, Instituto de Investigaciones Biológicas Clemente Estable, \\ Avenida Italia 3318, PC 11600 Montevideo, Uruguay \\ 2 Sección Entomología, Facultad de Ciencias, Iguá 4225, PC 11400 Montevideo, Uruguay \\ 3 Sección Etología, Facultad de Ciencias, Iguá 4225, PC 11400 Montevideo, Uruguay
}

Autor para correspondencia: mariana.c.trillo@gmail.com

\section{RESUMEN}

Guasuctenus longipes es una araña asociada a ambientes de quebrada. El objetivo de este estudio fue explorar la existencia de estructuras estridulatorias y emisión de sonido. Se describe el raspador y la lira en los palpos de los machos. Al tratarse de una especie nocturna, sugerimos que el canal acústico es preponderante para el encuentro de los sexos.

Palabras clave: Guasuctenus longipes, comunicación acústica, órganos estridulatorios

\section{ABSTRACT}

Zamba by you: possible stridulation during courtship in a spider with bridal veil (Ctenidae). Guasuctenus longipes is a spider associated with stream environments. The objective of this study was to explore the presence of stridulatory organs and sound emission. Scraper and ridges on the pedipalps of males were described. As it is a nocturnal species, we suggest that the acoustic channel is preponderant for the meeting of the sexes.

Keywords: Guasuctenus longipes, acoustic communication, stridulatory organs

El exoesqueleto duro de los artrópodos permite, entre otras funciones adaptativas, producir sonidos mediante el raspado o fricción de dos zonas, mecanismo conocido como estridulación (Uetz \& Stratton, 1982). Requiere el movimiento de una de las partes duras (raspador) sobre otra estructura (lira) (Joqué, 2005) y se transmite vibraciones por aire y/o sustrato (Uhl \& Elias, 2011). Son muchos los artrópodos que se comunican entre sí mediante sonidos, principalmente insectos (Kent, 1877), siendo menos conocida esta propiedad en los arácnidos. La estridulación se ha adquirido en varias instancias independientes durante la evolución, ya que ocurre en grupos diversos y los órganos estridulatorios presentan diferencias morfológicas y posicionales (Bristowe, 1929; Joqué, 2005; Witt \& Rovner, 2014). La producción de sonidos en arañas puede ocurrir en tres contextos diferentes: sonidos defensivos, agresivos o de cortejo (Uetz \& Stratton, 1982). Probablemente es más frecuente de lo que ha sido registrado debido a que generalmente ocurre a frecuencias inaudibles para el oído humano (Uhl \& Elias, 2011). La recepción de estas señales ocurre mediante ciertas setas especializadas que se encuentran en todo el cuerpo de la araña, principalmente en pedipalpos y patas (Foelix, 2011; Uhl \& Elias, 2011), conocidos como tricobotrias y sensilias (Barth, 2002).

Basándonos en la localización del raspador y/o la lira en el cuerpo de las arañas se conocen hasta el momento ocho tipos de órganos estridulatorios (Uhl \& Elias, 2011), Rovner (1975) los clasificó de la siguiente manera: 1. Raspado del abdomen sobre el cefalotórax (tipo a), o contra el pedicelo (tipo b); 2. Frotamiento de un apéndice sobre el otro, incluyendo quelíceroquelícero (tipo c), quelícero-pedipalpo (tipo d), pedipalpo-pata I (tipo e), pata I-pata II (tipo f); 3. Un apéndice frota contra el abdomen (tipo g); 4. El órgano estridulatorio (raspador y lira) se encuentra en superficies opuestas segmentarias de la unión de un mismo apéndice (tipo h). Los sonidos emitidos por frotamiento de cualquier par de partes duras fisiológicamente no tienen grandes posibilidades de ser modulados en intensidad ni frecuencia, pero sí en ritmo; son señales muy efectivas ya que producen altas frecuencias con movimientos de bajas frecuencias (Uhl \& Elias, 2011). Este tipo de señal puede 


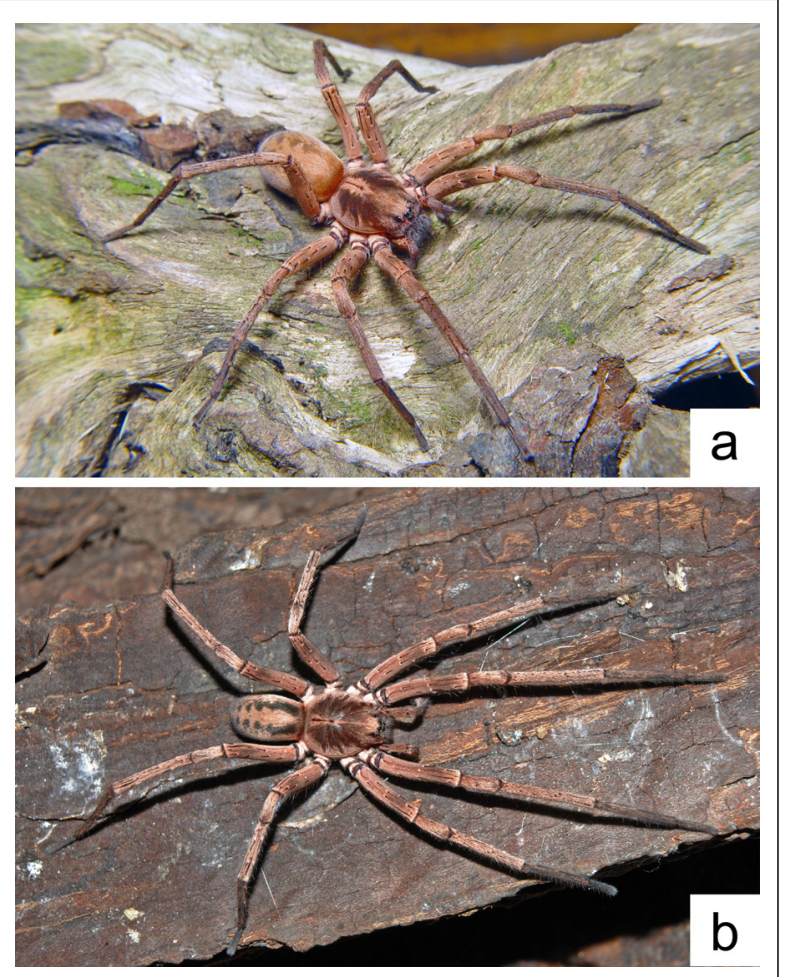

Fig. 1. Hembra adulta (a) y macho adulto (b) de Guasuctenus longipes.

propagarse por largas distancias, emitirse de día y de noche, y contienen una variedad de información potencial como frecuencia y amplitud (Uetz \& Stratton, 1982).

Guasuctenus longipes (Keyserling, 1891) es una especie de araña de la familia Ctenidae que se distribuye en Brasil y Uruguay (Polotow \& Brescovit 2019). En nuestro país se ha registrado en las localidades de Quebrada de los Cuervos (Treinta y Tres), Paso Centurión (Cerro Largo) y Valle del Lunarejo (Rivera). Es una especie descrita como prioritaria para la conservación en Uruguay (Ghione et al., 2017). Está asociada a montes de quebrada, valles profundos de gran pendiente que concluyen en cursos de agua, se trata de un ambiente particular de mucha humedad y poca luminosidad, en el que se forman dos grandes estratos verticales con diferentes especies de plantas (Grela \& Romero, 1997). Guasuctenus longipes se encuentra en el estrato cauce (más cercano al agua), entre las piedras y la vegetación herbácea. Los individuos de esta especie presentan el cefalotórax brillante, marrón rojizo, y el abdomen con una banda longitudinal amarillenta hacia el medio y dos bandas marrones a los lados (Fig. 1), patas con dos uñas y fascículos subungueales que les permiten trepar superficies lisas (Keyserling, 1891; Aisenberg et al., 2011). Presentan dimorfismo sexual, siendo las
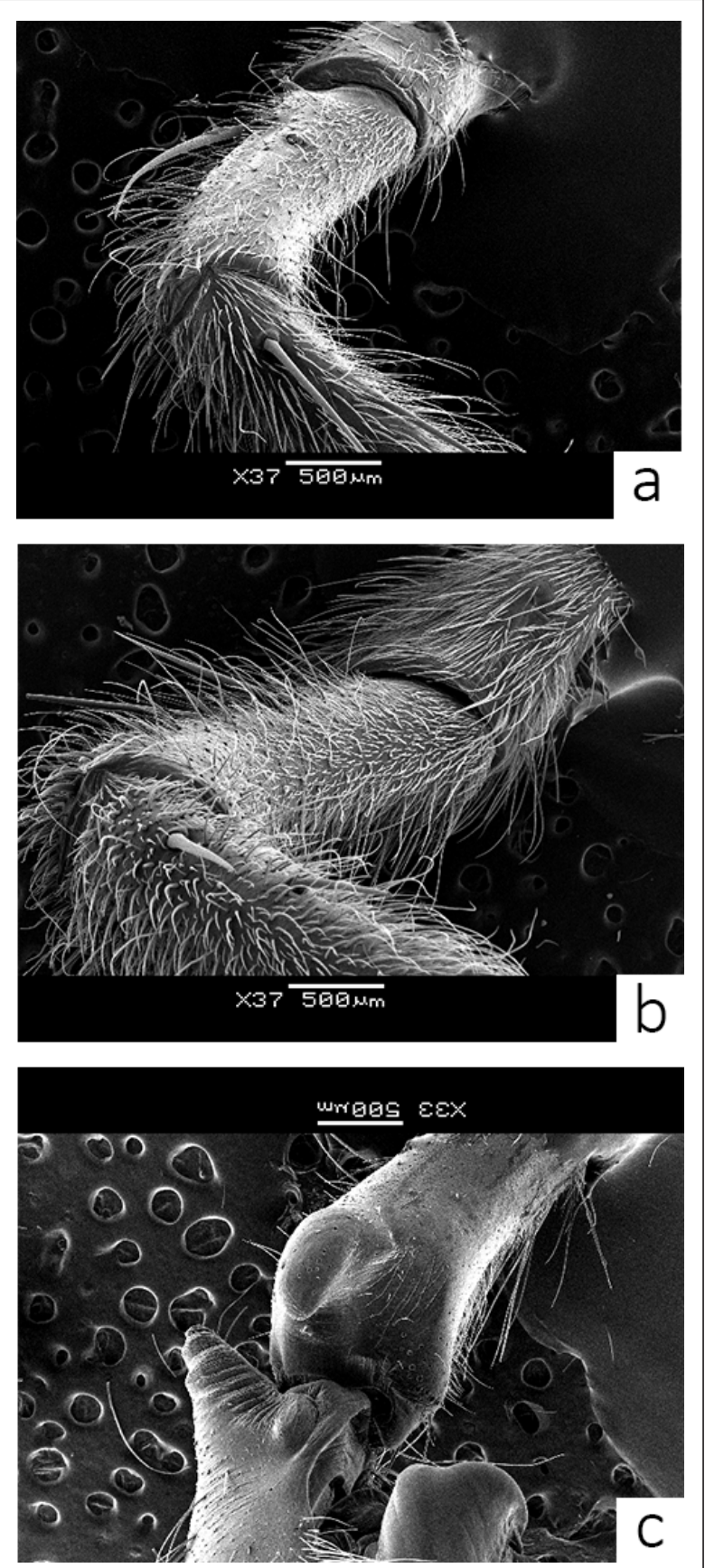

Fig. 2. Vista retrolateral de la articulación tibio-tarsal de los pedipalpos de Guasuctenus longipes, a) juvenil, b) hembra adulta, c) macho adulto.

hembras más grandes que los machos (Trillo et al., 2019). Durante la realización de experimentos de comportamiento sexual (Trillo et al., 2019), se pudo observar movimientos rítmicos de los pedipalpos por parte del macho, sugiriendo una posible estridulación como parte de la comunicación durante el cortejo, previo a la deposición de velo nupcial. El objetivo fue 
Tabla 1. Familias reportadas con capacidad de producción de sonidos mediante estridulación.

\begin{tabular}{|c|c|c|}
\hline Familia & Tipo de estridulación & Referencia bibliográfica \\
\hline Agelenidae & Estridulación tipo b & Uetz \& Stratton, 1982 \\
\hline Amaurobiidae & Estridulación & Uhl \& Elias, 2011 \\
\hline Anyphaenidae & Estridulación & Uhl \& Elias, 2011 \\
\hline Araneidae & Estridulación tipo g & Uetz \& Stratton, 1982 \\
\hline Archaeidae & Estridulación & Uhl \& Elias, 2011 \\
\hline Austrochilidae & Estridulación & Uhl \& Elias, 2011 \\
\hline Barychelidae & Estridulación tipo d & Uetz \& Stratton, 1982 \\
\hline Clubionidae & Estridulación tipo a & Uetz \& Stratton, 1982 \\
\hline Corinnidae & Estridulación tipo g & Ramírez et al., 2001 \\
\hline Ctenidae & Estridulación tipo h & Este trabajo \\
\hline Cyatholipidae & Estridulación & Uhl \& Elias, 2011 \\
\hline Diguetidae & Estridulación tipo d & Uetz \& Stratton, 1982 \\
\hline Dipluridae & Estridulación tipo d & Uetz \& Stratton, 1982 \\
\hline Gnaphosidae & Estridulación tipo a & Uetz \& Stratton, 1982 \\
\hline Hahniidae & Estridulación tipo a & Uetz \& Stratton, 1982 \\
\hline Leptonetidae & Estridulación & Uetz \& Stratton, 1982 \\
\hline Linyphiidae & Estridulación tipo d, f, g & Uetz \& Stratton, 1982 \\
\hline Lycosidae & Estridulación tipo g, h & Barth, 1982; Rovner, 1975;Uetz \& Stratton, 1982 \\
\hline Mecysmaucheniidae & Estridulación & Uhl \& Elias, 2011 \\
\hline Mimetidae & Estridulación tipo d & Uetz \& Stratton, 1982 \\
\hline Ochyroceratidae & Estridulación & Uetz \& Stratton, 1982 \\
\hline Palpimanidae & Estridulación & Uhl \& Elias, 2011 \\
\hline Pholcidae & Estridulación tipo d & Peretti et al., 2006 \\
\hline Phyxelididae & Estridulación & Uhl \& Elias, 2011 \\
\hline Salticidae & Estridulación tipo a, d, h & Elias et al., 2003; Uetz \& Stratton, 1982 \\
\hline Scytodidae & Estridulación tipo d & Uetz \& Stratton, 1982 \\
\hline Segestriidae & Estridulación & Uetz \& Stratton, 1982 \\
\hline Sicariidae & Estridulación tipo d & Uetz \& Stratton, 1982 \\
\hline Synotaxidae & Estridulación & Uhl \& Elias, 2011 \\
\hline Telemidae & Estridulación tipo b & Dupérré \& Tapia, 2015 \\
\hline Tetragnathidae & Estridulación tipo d & Jocqué, 2005 \\
\hline Theraphosidae & Estridulación tipos c, d, e & Uetz \& Stratton, 1982 \\
\hline Theridiidae & Estridulación tipos a, b & Uetz \& Stratton, 1982;Barth, 1982; Lee et al., 1986 \\
\hline Titanoecidae & Estridulación tipo h & Almeida-Silva et al., 2009 \\
\hline Uloboridae & Estridulación tipo d & Uetz \& Stratton, 1982 \\
\hline Zodariidae & Estridulación tipo d, e, f, g, h & Joqué, 2005 \\
\hline
\end{tabular}

analizar en detalle el cortejo en $G$. longipes y examinar los individuos con el fin de detectarla presencia de estructuras estridulatorias que pudieran estar implicadas en la comunicación entre los sexos.

Se utilizaron los individuos de G. Iongipes de Trillo et al. 2019. Los mismos fueron recolectados entre la vegetación, sobre los barrancos que rodean al arroyo Yerbal Chico de una zona cercana a la Quebrada de los Cuervos, Treinta y Tres, Uruguay (32055'39"S $54^{\circ} 27^{\prime} 25^{\prime \prime}$ ). Todos los ejemplares se encuentran depositados en la Colección de Entomología de Facultad de Ciencias, Universidad de la República (FCE-Ar 6427-6444).

Con el fin de corroborar la existencia de órganos estriduladores en $G$. longipes se revisaron ejemplares bajo una lupa estereoscópica Olympus 0-015 y se 

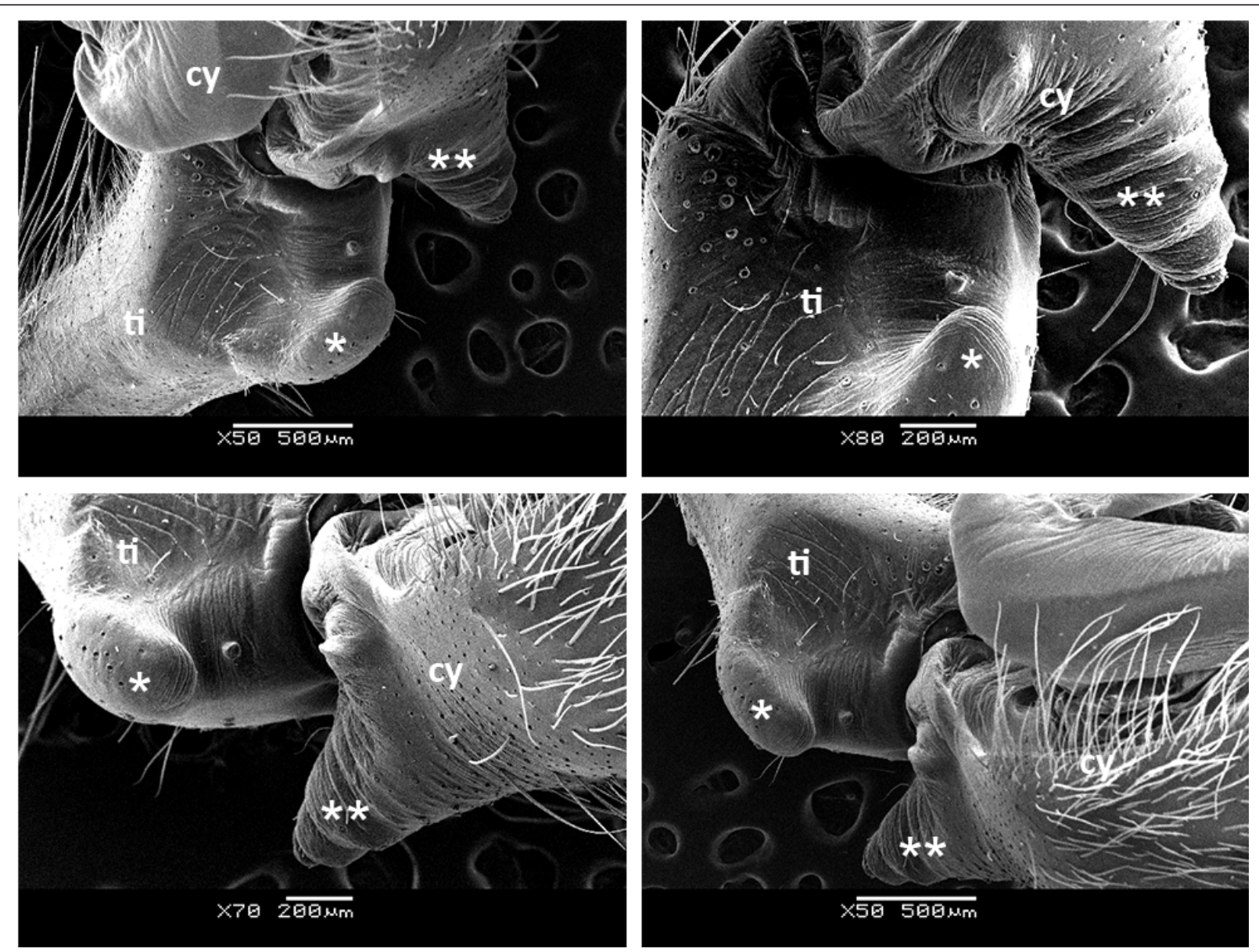

Fig. 3. Close up de la vista retrolateral de los órganos estridulatorios palpares del macho de Guasuctenus longipes, ti = tibia, cy $=$ cymbium; ${ }^{*}=$ lira, ${ }^{* *}=$ raspador.

tomaron fotografías de los pedipalpos de juveniles, hembras y machos adultos con el microscopio electrónico de barrido SEM Jeol JSM-5900LV del Servicio de Microscopía, Facultad de Ciencias, Montevideo, Uruguay. Se analizó en detalle la existencia de movimientos del tipo estridulatorio a través de los videos de Trillo et al. $2019(\mathrm{n}=11)$ mediante el programa J- Watcher (Blumstein et al. 2000).

Como resultados, se identificaron posibles estructuras estridulatorias de raspador y lira exclusivamente en machos adultos (Fig. 2), localizadas en la unión tibio-tarsal de los pedipalpos; específicamente, la lira se encontró en la parte distal de la tibia (base de la RTA) y el raspador en la parte dorsal del cymbium (Fig. 3). Además, se observó un posible movimiento de raspado de los pedipalpos que consiste en el movimiento alternado de palpos hacia arriba y abajo, oscilando sus segmentos distales $(\mathrm{n}=$ 9; número de apariciones $=4,89 \pm 5,67$; duración= 4.26 \pm 7.74 segundos; máx.=18, $\min .=0$ ), (disponible en: https://youtu.be/qhOhwHNj2ss).
Este es el primer reporte de órganos estridulatorios y su uso durante del cortejo en arañas de la familia Ctenidae (Tabla 1); corresponderían al tipo h de Rovner (1975). Es posible que existan más especies con órganos estridulatorios señalados en sus descripciones originales, pero no se ha abordado aún el estudio de su función comportamental (M. Simó com. pers.). La presencia de estructuras estridulatorias exclusivamente en machos adultos y los cambios de dirección de las hembras luego del comportamiento asociado a la posible estridulación, sugiere que estos órganos tienen una función comunicativa durante el cortejo, posiblemente para aumentar la receptividad femenina o facilitar el encuentro (Foelix, 2011). La estridulación es una señal que se propaga a largas distancias y no se ve tan afectada por clima o la luz como ocurre con otras señales (Uhl \& Elias, 2011).

Asimismo, G. longipes es una araña de actividad nocturna y se la ha encontrado en variados sustratos: sobre la tierra, vegetación, e incluso cerca o lejos de cursos de agua de diferentes localidades de Uruguay 
(Simó \& Brescovit, 2001). El uso de este canal comunicativo es muy eficiente en diferentes ambientes, por lo que podría funcionar como reforzador de reconocimiento individual o específico (Eberhard, 1996; Joqué, 2005). Esto concuerda con la hipótesis de "Mate check", que asume que la cantidad de información transmitida en el cortejo se relaciona con la especialización ecológica de la especie (Joqué, 2005). Los machos de G. longipes realizaron estridulaciones antes del contacto, fase acústica en la que parecería que se orientan entre sí. Al tratarse de una especie nocturna, se podría esperar que el canal acústico sea preponderante para el encuentro y la sincronización de la receptividad en ambos sexos. En la zona de la Quebrada de los Cuervos, esta especie vive en simpatría con otros cténidos de los géneros Parabatinga y Asthenoctenus, dos especies de menor tamaño que $G$. longipes. Sin embargo, en otras zonas de su distribución (por ejemplo, Brasil), estas arañas viven en simpatría con varias especies de la misma familia y de tamaño similar, por lo que en esas zonas sí resultarían importantes los reforzamientos de señales a modo de barreras reproductivas.

Futuros experimentos se focalizarán en confirmar la emisión de sonido durante el cortejo en esta especie mediante grabaciones acústicas y realización de sonogramas.

Agradecemos a M. González por su ayuda en el uso del programa J-Watcher, R. Postiglioni por su ayuda con la bibliografía. También agradecemos a $\mathrm{E}$. Stanley, A. Albín, F. Baldenegro, M. Carballo y L. Montes de Oca por su colaboración en la cría de individuos. Agradecemos a M. Simó por su contribución con Bibliografía y acceso al material depositado en Colección de Entomología de Facultad de Ciencias, Montevideo, Uruguay. A.A. y G.F. agradecen el soporte de PEDECIBA, UdelaR, y SNI (ANII). Los autores agradecen al editor y dos revisores anónimos por sus valiosos aportes al manuscrito.

\section{BIBLIOGRAFÍA}

Aisenberg A., Toscano-Gadea C. \& S. Ghione. 2011. Guía de Arácnidos del Uruguay. Ediciones de la fuga. Colección Ciencia Amiga. 256 pp.

Almeida-Silva L.M., Brescovit A.D. \& C.E. Griswold. 2009. On the poorly known genus Anuvinda Lehtinen, 1967 (Araneae: Titanoecidae). Zootaxa, 2266: 61-68.

Barth F.G. 2002. A spider's world. Senses and behavior. Springer Verlag Berlin Heidelberg New York. $394 \mathrm{pp}$.

Blumstein D.T., Daniel J.C. \& C.S. Evans. 2000. JWatcher. Version 0.9. Available in http:// www.jwatcher.ucla.edu/

Bristowe B.A. 1929. The mating habits of spiders, with special reference to the problems surrounding sex dimorphism. Proceedings of the Zoological Society of London, 11: 309-356.

Duperre N. \& E. Tapia. 2015. Discovery of the first telemid spider (Araneae, Telemidae) from South America, and the first member of the family bearing a stridulatory organ. Zootaxa, 4020(1): 191-196.

Eberhard W.G. 1996. Female Control: sexual selection by cryptic female choice. Princeton University Press. Princeton, New Jersey. 502 pp.

Elias D.O., Mason A.C., Maddison W.P. \& R.R. Hoy. 2003. Seismic signals in a courting male jumping spider (Araneae: Salticidae). Journal of Experimental Biology, 206: 4029-4039.

Foelix R.F. 2011. Biology of Spiders. Oxford University Press. UK. 419 pp.

Ghione S., Coelho L., Costa F.G., García L.F., González M., Jorge C., Laborda Á., Montes de Oca L., Pérez-Miles F., Postiglioni R., Simó M., ToscanoGadea C., Viera C. \& A. Aisenberg. 2017. Arácnidos prioritarios para la conservación en Uruguay. Boletín de la Sociedad Zoológica del Uruguay, 26 (1): 1-8.

Grela I. \& M.F. Romero. 1997. Estudio comparativo en dos sectores de bosques de quebrada del arroyo Lunarejo, Departamento de Rivera. Tesis Ingeniero Agrónomo. 80 pp.

Joqué R. 2005. Six stridulating organs on one spider (Araneae, Zodariidae): is this the limit? Journal of Arachnology, 33: 597-603.

Kent W.S. 1877. Sound-producing arthropods. Nature, 17 (418): 11-11.

Lee R.C.P., Nyffeler M. \& E. Krelina. 1986. Accoustic communication in two spider species of the genus Steatoda (Araneae, Theridiidae). The Journal of the Swiss Entomological Society, 59: 337-348.

Peretti A., Eberhard W.G. \& R.D. Briceño. 2006. Copulatory dialogue: female spiders sing during copulation to influence male genitalic movements. Animal Behavior, 72: 413-421.

Polotow D. \& A.D. Brescovit. 2019. Guasuctenus gen. nov., a new Neotropical spider genus of Ctenidae (Araneae). Zootaxa, 4624(4): 539-550.

Ramírez M.J., Lopardo L. \& A.B. Bonaldo. 2001. A review of the Chilean spider genus Olbus, with notes on the relationships of the Corinnidae (Arachnida, Araneae). Insect systematics and evolution, 31: 441-462.

Redondo T. 1994. Comunicación: teoría y evolución de las señales. En Carranza J. (ed.) Etología: Introducción a la Ciencia del Comportamiento. Publicaciones de la Universidad de Extremadura. Cáceres, España, 255-297.

Rovner J.S. 1975. Sound production by neartic wolf 
spiders: a substratum-coupled stridulatory mechanism. Science, 190: 1309-1310.

Simó M. \& A.D. Brescovit. 2001. Revision and cladistic analysis of the Neotropical spider genus Phoneutria Perty, 1833 (Araneae, Ctenidae), with notes on related Cteninae. Bulletin of the British Arachnological Society, 12 (2): 67-82.

Trillo M.C., Laborda Á., Francescoli G. \& A. Aisenberg. 2019. Fifty shades of silk: sexual behavior and bridal veil deposition in the spider Ctenus longipes. Actaethologica, 22(1): 47-56.

Uetz G.W. \& G.E. Stratton. 1982. Acoustic Communication and reproductive isolation in spiders. En: Witt P.N. \& Rovner J.S. (eds.). Pp.
123-159. Spider Communication: Mechanism and Ecological Significance. Princeton University Press. Princeton, New Jersey.

Uhl G. \& D.O. Elias. 2011. Communication. En: Herberstein M. Spider Behaviour. Flexibility and Versatility. Pp. 127-189. Cambridge University Press. Macquarie University, Sydney, Australia.

Witt P.N. \& J.S. Rovner. 2014. Spider communication: mechanisms and ecological significance. Princeton University Press. 536 pp.

Fecha de Recepción: 15 de septiembre de 2020 Fecha de Aceptación: 17 de diciembre de 2020 